\title{
Integrated Microsystem
}

\author{
for \\ 3D Magnetic Field Measurements
}

\author{
Piero Malcovati and Franco Maloberti \\ University of Pavia
}

\begin{abstract}
In this paper we present a portable battery-operated 3D magnetic microsystem (magnetodosimeter), intended for monitoring the workers' exposure to magnetic fields, in particular, working environments such as hospitals or physics laboratories. The proposed microsystem is based on a multi-chip module containing three equal channels for the three components of the magnetic field measurement, a microprocessor and a memory. Each single-chip channel detects the magnetic field, converts it into the digital domain and delivers the result to the microprocessor by means of an on-chip serial interface. The single-chip channel, fabricated in a $0.8 \mu \mathrm{m}$ CMOS technology, is sensitive to magnetic fields ranging from $-200 \mathrm{mT}$ to $+200 \mathrm{mT}$, achieving 12 bits of resolution and 11 bits of linearity.
\end{abstract}

\section{INTRODUCTION}

Several working environments, such as hospitals (magnetic resonance) or physics laboratories (nuclear experiments) are polluted by strong low-frequency

Authors' Current Addresses:

P. Malcovati, Department of Electrical Engineering, University of Pavia, Via

Ferrata 1, 27100 Pavia, Italy; and F. Maloberti, Department of Electronics,

University of Pavia, Via Ferrata 1, 27100 Pavia, Italy.

Based on a presentation at IEEE 1999 IMT.

0885/8985/99/\$10.00 1999 IEEE magnetic fields. In order to guarantee the workers' health and protect the employers against legal action, therefore, it is essential to monitor the integral exposure of each worker to a particular level of field. In order to accomplish this task, inexpensive, battery-operated magnetodosimeters and such magnetic field sensitive badges are required. These devices must monitor the module of the magnetic field vector in the environment during a whole working shift and store a histogram of the magnetic field levels. Moreover at the end of the working day, the data stored in the magnetodosimeter must be dumped on a PC.

The level of magnetic field known to be dangerous for human beings can be detected by silicon magnetic sensors fabricated in conventional CMOS technologies, such as Hall devices [1]. It is, therefore, possible to realize, at low cost, a single chip containing both the magnetic sensor and the interface circuits required to detect a single component of the magnetic field. In this paper we present an integrated microsystem for magnetic field measurements, specifically designed to be the core of a miniaturized magnetodosimeter. The proposed device detects the magnetic field in the environment, converts it into the digital domain, and delivers the result to a microprocessor by means of an on-chip serial interface.

\section{MICROSYSTEM ARCHITECTURE}

The most important requirements of the magnetodosimeter and, hence, of the single-chip channel are summarized in Table 1, on next page. In order to cover 
Table 1. Required Performance of the 3D Magnetic Sensing Microsystem

\begin{tabular}{|l|c|}
\hline \multicolumn{1}{|c|}{ Parameter } & Value \\
\hline Magnetic Field Range & $\pm 200 \mathrm{mT}$ \\
\hline Resolution & $\begin{array}{l}100 \mu \mathrm{T} \\
12 \mathrm{Bits}\end{array}$ \\
\hline Power Supply Voltage & $5 \mathrm{~V}$ \\
\hline Magnetic Field Sampling Frequency & $1 / 32 \mathrm{~Hz} \div 1 \mathrm{~Hz}$ \\
\hline Recording Time & $8 \mathrm{~h}$ \\
\hline Memory (1 S/s, $8 \mathrm{~h}$ ) & $4 \mathrm{~KB}$ \\
\hline Microprocessor Interface & Serial \\
\hline
\end{tabular}

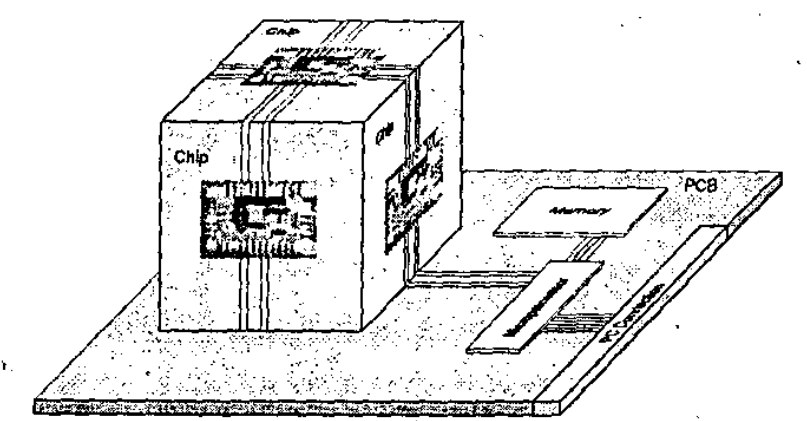

Fig. 1. Mechanical Arrangement of the 3D Magnetic Sensing Multi-Chip Module .

the magnetic field range from $-200 \mathrm{mT}$ to $+200 \mathrm{mT}$ with steps of $100 \mu \mathrm{T}$ at least $11+1$ bits (2048 levels and a sign bit) are required. However, since the sign of the magnetic field is not relevant in this application, only the least significant bits are stored in the RAM. Hence, the size of the memory required to monitor the magnetic field once per second during a whole working shift is 4 KBytes.

Figure 1 shows the complete magnetodosimeter. The system is based on a multi-chip module containing three equal channels for the three components of the magnetic field $\left(\mathbf{B}_{\mathbf{x}}, \mathrm{B}_{\mathbf{y}}\right.$, and $\left.\mathrm{B}_{\mathrm{z}}\right)$, a microprocessor and a memory.

Each single-chip channel consists of a Hall device with front-end circuitry, an instrumentation amplifier, an incremental $\mathrm{A} / \mathrm{D}$ converter with the required digital circuitry and a serial bus interface, which interconnects the three modules with the microprocessor, as shown in Figure 2.

The magnetic sensor used is a spinning current Hall device [2], which produces an output voltage proportional to the magnetic field component perpendicular to the chip with very low offset. The sensitivity of this particular device, equal to $200 \mu \mathrm{V} /(\mathrm{mA} \mathrm{mT})$, can be trimmed by properly setting the bias current, in order to achieve the required accuracy in the whole system.

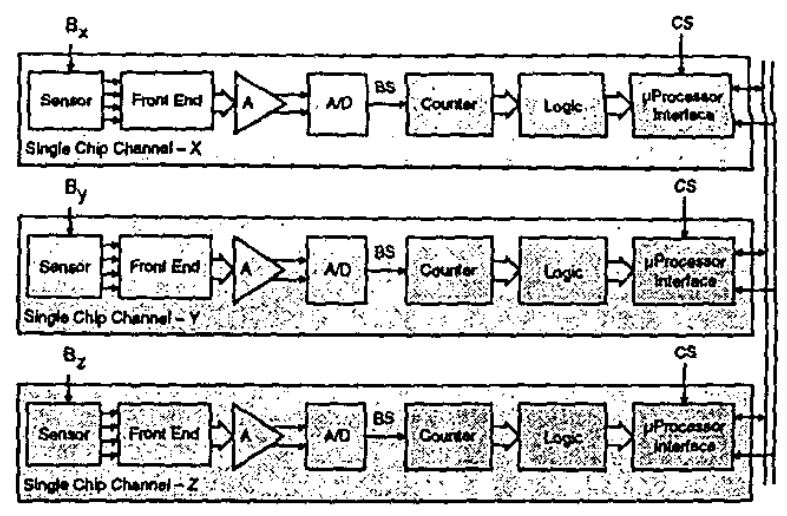

Fig. 2. Electrical Block Diagram of the 3D Magnetic Sensing Microsystem

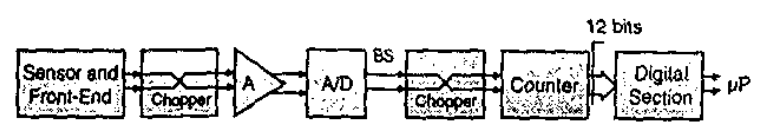

Fig. 3. Block Diagram of the Interface Circuit for the Single Channel of the 3D Magnetic Sensing Microsystem

\section{INTERFACE CIRCUIT DESCRIPTION}

The signal obtained at the output of the sensor over the considered magnetic field range is in the order of few tens of $\mathrm{mV}$, thus requiring low-noise and low-offset amplification before A/D conversion. In order to achieve the required noise and offset performance in CMOS technology, we used chopper stabilization, as shown in Figure 3. The sensor output signal, modulated with a square wave, is shifted in a region of the spectrum where the noise of the instrumentation amplifier and of the $A / D$ converter is dominated by the thermal component. After amplification and A/D conversion, then, the signal is modulated again in the digital domain and shifted back in the original band. The offset and the large low frequency noise of the instrumentation amplifier and the A/D converter (flicker noise) are also modulated and, hence, pushed at high frequency, where they are removed by a subsequent digital low-pass filter (Counter).

\section{Instrumentation Amplifier}

The schematic of the used high-input-impedance instrumentation amplifier is shown in Figure 4, on next page. The resistive feedback provides a gain of $10\left(R_{1}=\right.$ $54 \mathrm{~K} \Omega$ and $R_{2}=12 \mathrm{~K} \Omega$ ), while the cross-coupled switches connected to the input allow us to implement the chopper stabilization.

The operational amplifiers used are based on a folded cascode structure followed by a low-impedance output buffer, which drives the resistive feedback. 


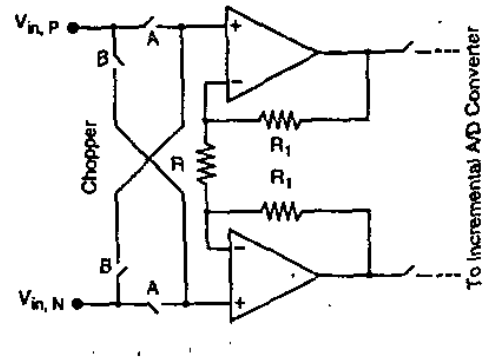

Fig. 4. Schematic of the Front-End Instrumentation Amplifier

Table 2. Capacitance and Parameter Values of the 12-Bit Incremental A/D Converter

\begin{tabular}{|l|c|}
\hline \multicolumn{1}{|c|}{ Capacitor } & Value \\
\hline Integrating Capacitor $\mathrm{C}_{\mathrm{I}}$ & $2 \mathrm{pF}$ \\
\hline Input Capacitor $\mathrm{C}_{\mathrm{in}}$ & $2 \mathrm{pF}$ \\
\hline Reference Capacitor $\mathrm{C}_{\mathrm{R}}$ & $2 \mathrm{pF}$ \\
\hline Sample and Hold Capacitor $\mathrm{C}_{\mathrm{S}}$ & $2 \mathrm{pF}$ \\
\hline Parameter & Value \\
\hline Clock Frequency & $1 \mathrm{MHz}$ \\
\hline
\end{tabular}

\section{Incremental $\mathbf{A} / \mathbf{D}$ Converter}

The output signals of the instrumentation amplifier are directly connected to the input of an incremental A/D converter, which allows high resolution (in this case, 12 bits) to be achieved with a simple hardware and a low power consumption.

The schematic of the switched-capacitor incremental A/D converter [3,4] is shown in Figure 5, while the capacitance and design parameter values are summarized in Table. 2. This circuit, consisting of an integrator with autozero [5] and a latched comparator, is very similar to a first order sigma-delta modulator. The integrator, however, is reset at the end of each conversion cycle $\left(\phi_{R}\right)$, consisting of $2^{N}$ clock periods, where $N$ is the required resolution.

Because of the periodic reset of the integrator, the behavior of this circuit is deterministic rather than stochastic (i.e., for equal input signals we obtain equal output bitstreams).

Moreover, the decimating filter can be reduced to a simple up/down counter, as shown in Figure 2. The incremental conversion algorithm is described by

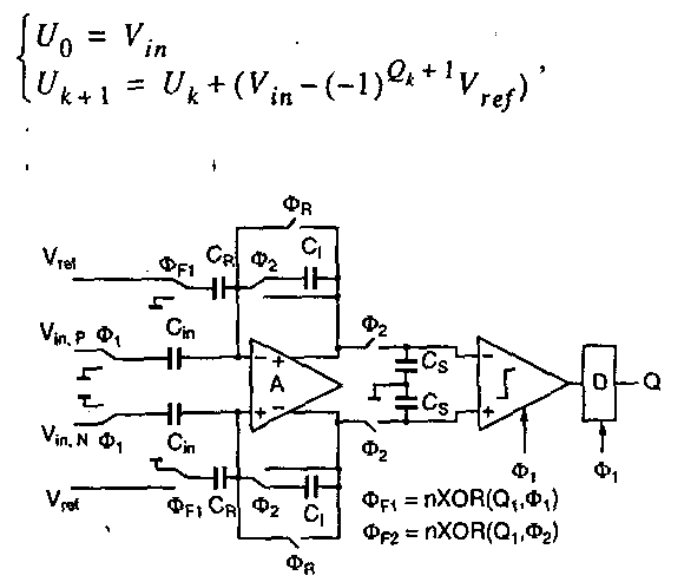

Fig. 5. Schematic of the 12-Bit Incremental A/D Converter

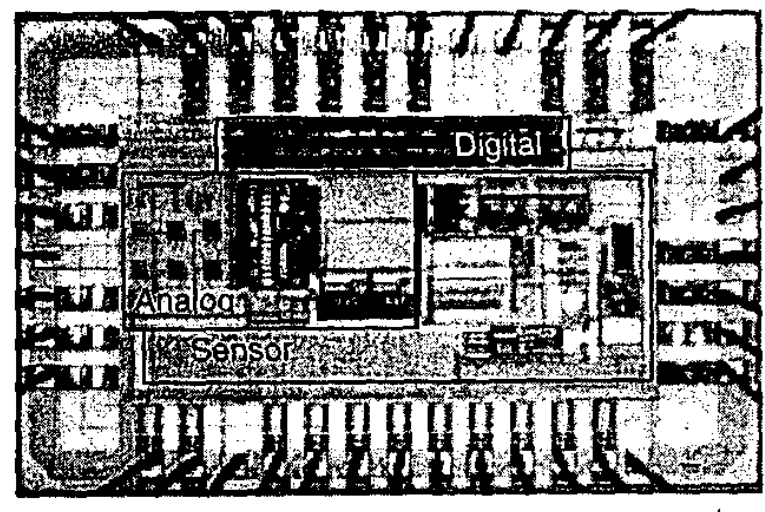

Fig. 6. Micrograph of a Single Channel of the 3D Magnetic Sensing Microsystem

where $\mathrm{U}$ and $\mathrm{Q}$ denote the output signals of the integrator and the comparator, respectively, while $k$ denotes the current clock period. The $\mathrm{N}$ bit digital output signal obtained after $2^{\mathrm{N}}$ clock periods is, therefore, given by

$$
\text { Out }=\sum_{i=0}^{N-1} Q_{i}=\operatorname{Round}\left(2^{N} \frac{V_{i n}}{V_{\text {ref }}}\right)
$$

The operational amplifier used is based on a fully-differential, folded-cascode structure with switched-capacitor common-mode feedback [6], while the latched-comparator consists of a differential stage with double regenerative loop, followed by an output latch [7]. The incremental A/D converter is operated with a clock frequency of $1 \mathrm{MHz}$.

\section{Digital Section}

The digital section of the proposed microsystem consists of a chopper modulator (multiplexer), an up/down counter for decimating the output bitstream of the incremental A/D converter, the logic required for controlling the operation of the incremental A/D converter 


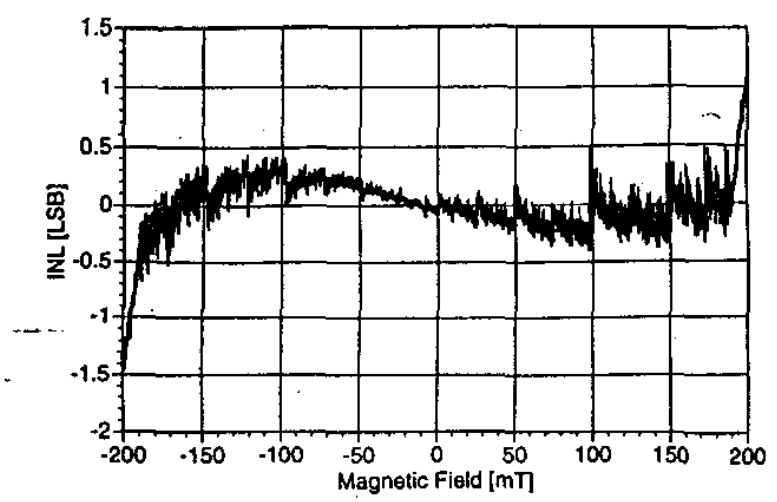

Fig. 7. Measured Integral Nonlinearity of the 3D Magnetic Sensing Microsystem

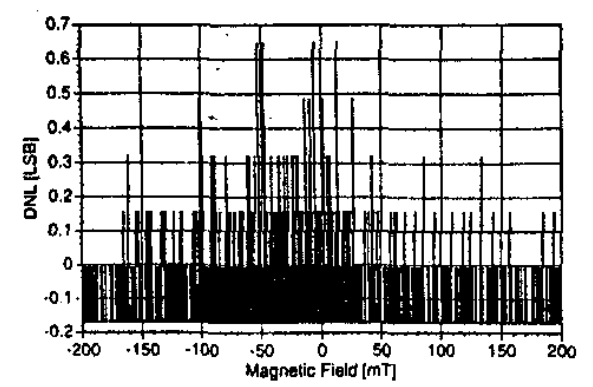

Fig. 8. Measured Differential Nonlinearity of the 3D Magnetic Sensing Microsystem

and, finally, a serial interface for interconnecting the three channels of the magnetodosimeter with the microprocessor.

The serial interface implements a standard two-wire (Data and Clock) communication protocol between A/D converters and microprocessors. The microprocessor provides a chip select signal (CS) which enables the A/D converter. After $2^{\mathrm{N}}$ clock cycles, when the data are ready, the A/D converter produces an end-of-conversion signal on the data line. The microprocessor, then, provides a clock signal on the clock line and reads the data serially on the data line.

When the chip is not selected $(\overline{\mathrm{CS}})$ the whole system enters in power-down mode (the analog blocks, including the sensor are switched off), in order to reduce the average power consumption. In fact, if the magnetic field sampling frequency is set to $1 \mathrm{~Hz}$, the system is operated with a $0.4 \%$ duty-cycle.

\section{EXPERIMENTAL RESULTS}

A prototype of the single channel chip has been integrated in a standard $0.8 \mu \mathrm{m}$ CMOS process. The total die area, including pads is $3.2 \mathrm{~mm} \times 2.1 \mathrm{~mm}$.

The measured integral (INL) and differential (DNL) nonlinearities of the whole system are shown in Figure 7 and Figure 8, respectively. The obtained results lead to an effective resolution of 12 bits with 11 bits of linearity (without calibration). The slight degradation in the linearity performance can be corrected digitally by the microprocessor if required, although in the considered application 11 bits of linearity are sufficient.

\section{CONCLUSIONS}

In this paper we presented a portable battery-operated microsystem (magnetodosimeter) sensitive to 3D magnetic fields, ranging from $-200 \mathrm{mT}$ to $+200 \mathrm{mT}$. The system is based on a multi-chip module containing three equal channels for the three components of the magnetic field, a microprocessor and a memory. Each single-chip channel consists of a spinning current Hall device with front-end circuitry, an instrumentation amplifier, an incremental A/D converter and a digital section. Chopper stabilization is used to cancel the offset of the system. An on-chip two-wire serial interface allows the communication between the three channels of the magnetodosimeter and the microprocessor. The single-chip channel, fabricated in a $0.8 \mu \mathrm{m}$ CMOS technology achieves 12 bits of resolution and 11 bits of linearity.

\section{ACKNOWLEDGMENTS}

This work was partially supported by the E.U. Esprit Project 20360 MagIC. The authors wish to acknowledge Austria Mikro Systeme (AMS) and, in particular, Dr. T. Olbrich for providing the used Hall sensors.

\section{REFERENCES}

[1] H. Baltes and R. Castagnetti, 1994, Magnetic Sensors, in S.M. Sze, ed., Semiconductor Sensors, Wiley, New York, NY, pp. 205-269.

[2] S. Bellekorn and P. Munter, 1993, Offset Reduction in Spinning Current Hall Plates, Sensors and Materials, Vol. 5, pp. 253-260.

[3] J. Robert, G.C. Temes, V. Valencic, R. Dessoulavy and Ph. Deval, 1987,

A 16-bit Low Voltage A/D Converter, IEEE J. Solid-State Circuits, Vol. 22, pp 157-163.

[4] Ä. Häberli, M. Schneider, P. Malcovati, R. Castagnetti, F. Maloberti and $\mathrm{H}$. Baltes, 1996,

2D Magnetic Microsensor with OnChip Signal Processing for Contactless Angle Measurement, IEEE J. Solid-State Circuits, Vol. 31, pp. 1902-1907.

[5] G.C. Temes and K. Haug, 1984, Improved Offset Compensated Schemes for Switched-Capacitor Circuits, Electron. Lett., Vol. 20, pp. 508-509.

[6] P. Malcovati, 1996,

CMOS Thermoelectric Sensor Interfaces, Ph.D. Thesis, No. 11424, ETH Zurich, Zurich, Switzerland.

[7] G.M. Yin, F. Op't Einde and W. Sansen, 1992 A High-Speed Comparator with 8-b Resolution, IEEE J. Solid-State Circuits, Vol. 27, pp. 208-211. 